\title{
FINITE DETERMINATION ON ALGEBRAIC SETS
}

\author{
L. KUSHNER
}

\begin{abstract}
The concept of finite relative determination was introduced by Porto and Loibel [P-L] in 1978 and it deals with subspaces of $\mathbf{R}^{n}$. In this paper we generalize this concept for algebraic sets, and relate it with finite determination on the right. We finish with an observation between Lojasiewicz ideals and finite relative determination.
\end{abstract}

\section{INTRODUCTION}

We shall denote by $\mathscr{E}(n)$ the R-algebra of germs of differentiable maps and, by $\mathfrak{m}(n)$ its maximal ideal, and by $\mathbf{R}[x]$ the $\mathbf{R}$-algebra of polynomials with coefficients in $\mathbf{R}$. If $f$ is a germ, $j^{m} f(0)$ will denote the Taylor expansion up to degree $m$ of $f$ around the origin, and $\langle d f\rangle$ will denote the ideal of $\mathscr{E}(n)$ generated by $\partial f / \partial x_{j}$, the partial derivatives of $f$. If $j^{q}(n, 1)$ denotes the space of $q$-jets, then $\pi_{q}: \mathscr{E}(n) \rightarrow j^{q}(n, 1)$ is the canonical map which assigns $j^{q} f(0)$ to each $f$.

Let $S$ be a germ of a subset of $\mathbf{R}^{n}$ containing the origin and $J$ the ideal of germs which vanish at $S$. Let $G_{S}$ be the subgroup of diffeomorphisms which are the identity on $S$. Let $f$ and $g$ be germs such that $j^{k} g(0)=j^{k} f(0)$ and $f-g \in J$. We want to give necessary and sufficient conditions to show that $g$ is in the $G_{S}$ orbit of $f$.

The works of Mather [M] and Porto-Loibel [P-L] solve the case for $S$ the set of zeros of the ideals $\left\langle x_{1}, \ldots, x_{n}\right\rangle$ and $\left\langle x_{1}, \ldots, x_{s}\right\rangle$ respectively. In this work we solve the case for more general algebraic sets (Theorem 16), for example, the set $x^{2}-y^{3}=0$. We also give two theorems (Theorems 19 and 20) relating finite determinacy on the right and finite determinacy with respect to $G_{S}$ for a particular algebraic set $S$ which generalizes Theorem 1.10 of [P-L]. We finish with a theorem relating Lojasiewicz's ideals and finite relative determination.

Let $S$ be a germ of a subset of $\mathbf{R}^{n}$ containing the origin and $J$ the ideal of germs that are zero in $S$. We consider $\mathscr{L}$, the ideal of germs of vector fields whose coordinates belong to $J$. If $\phi_{t}$ is a one-parameter group germ for $X$ in $\mathscr{L}$, then $\phi_{t}$ restricted to $S$ is Id, the identity map.

Let $G_{S}$ be the group of germs of diffeomorphisms of $\mathbf{R}^{n}$ such that the identity is restricted to $S$.

Theorem 0. The tangent space of $G_{S}$ at the identity is $\mathscr{L}$.

Received by the editors October 9, 1989 and, in revised form, December 20, 1989.

1980 Mathematics Subject Classification (1985 Revision). Primary 58C27; Secondary 14B05. 
Proof. Let $X \in \mathscr{L}$ and consider $\phi_{t}$, the one-parameter group of $X$; it is clear that $\phi_{0}=\mathrm{Id}, \phi_{t} \in G_{S}$, and $\frac{\partial}{\partial t} \phi_{t}(x)=X \circ \phi_{t}(x)$; if we set $t=0$ we get $\left.\frac{\partial}{\partial t} \phi_{t}(x)\right|_{t=0}=X(x) \in T_{\text {Id }} G_{S}$.

Conversely, given $v \in T_{\mathrm{Id}} G_{S}$, there exists $\gamma: I \rightarrow G_{S}$ with $\gamma(0)=$ Id and $\dot{\gamma}(0)=v$. Since $\gamma(t) \in G_{S}$ it follows that $\gamma(t)(x)=x \forall x \in S$. Then $\dot{\gamma}(0)(x)=$ $0 \forall x \in S$ and $v$ is zero in $S$.

Definition 1. Let $f \in \mathfrak{m}(n)$. We say $f$ is $k$-determined relative to $G_{S}$ if given $g$ such that $j^{k} f(0)=j^{k} g(0)$ and $f-g \in J$, there exists $\phi \in G_{S}$ such that $g=f \circ \phi$.

We state without proof:

Theorem 2. Let $t_{0} \in \mathbf{R}$ be fixed, let $f$ and $g$ be in $\mathfrak{m}(n)$ with $\left.f\right|_{S}=\left.g\right|_{S}$, and let $F: \mathbf{R}^{n} \times \mathbf{R} \rightarrow \mathbf{R}$ be given by $F(x, t):=F_{t}(x)=(1-t) f(x)+\operatorname{tg}(x)$. Then the following assertions are equivalent:

(A) There exists a germ $H: \mathbf{R}^{n} \times \mathbf{R} \rightarrow \mathbf{R}^{n}$ such that

(1) $H(x, t)=x ; t \sim t_{0}, x \sim 0, x \in S$,

(2) $H_{t_{0}}=$ Id ,

(3) $F_{t} \circ H_{t}=F_{t_{0}} ; t \sim t_{0}$,

where $\sim$ means near $t_{0}$.

(B) There exists a germ $h: \mathbf{R}^{n} \times \mathbf{R} \rightarrow \mathbf{R}^{n}$ such that

(I) $\sum_{i=1}^{n} \frac{\partial F}{\partial x_{i}}(x, t) h_{i}(x, t)+\frac{\partial F}{\partial t}(x, t)=0 ; t \sim t_{0}$,

(II) $h_{i}(x, t)=0 ; t \sim t_{0}, x \sim 0, x \in S$,

where $h=\left(h_{1}, h_{2}, \ldots, h_{n}\right)$.

Observation. Let

$$
\sum_{i=1}^{n} \frac{\partial F}{\partial x_{i}}(h(t, x), t) \frac{\partial h_{i}}{\partial t}(x, t)+\frac{\partial F}{\partial t}(h(t, x), t)=0,
$$

where $H=\left(h_{1}, \ldots, h_{n}\right)$. Then (1), (2), (3) are equivalent to (1), (2), (3').

Definition 3. Let $I$ be an ideal of $\mathbf{R}[x]$, the ring of polynomials in $x_{1}, \ldots, x_{n}$ variables, let $z(I)=\left\{x \in \mathbf{R}^{n} \mid f(x)=0 \forall f \in I\right\}$, and suppose $0 \in z(I)$. Then $\widehat{I}=\left\{f \in \mathscr{E}(n)|f|_{z(I)} \equiv 0\right\}$. We say $I$ is radical if $I=\widehat{I}$.

Some examples. (1) If $I=\left\langle x_{1}, \ldots, x_{s}\right\rangle$, then $\widehat{I}$ is generated by $\left\{x_{1}, \ldots, x_{s}\right\}$.

(2) If $I=\left\langle x_{i} x_{j}\right\rangle_{1 \leq i \leq s}^{n-t+1 \leq j \leq n}$ with $s+t \leq n$, then $\widehat{I}$ is generated by $\left\{x_{i} x_{j}\right\}_{1 \leq i \leq s}^{n-t+1 \leq j \leq n}$.

(3) If $I=\left\langle x_{1} x_{2}, x_{1} x_{3}, x_{2} x_{3}\right\rangle, n=3$, then $\hat{I}=I$.

In (3) it is clear that $z(I)=\mathbf{R} \times\{0\} \times\{0\} \cup\{0\} \times \mathbf{R} \times\{0\} \cup\{0\} \times\{0\} \times \mathbf{R}$. By Hadamard's lemma we get for $f$ in $\widehat{I}$ :

$$
f\left(x_{1}, x_{2}, x_{3}\right)=x_{1} x_{2} g_{12}+x_{1} x_{3} g_{13}+x_{2} x_{3} g_{13}+x_{1}^{2} g_{11}+x_{2}^{2} g_{22}+x_{3}^{2} g_{33} \text {. }
$$

Now

$$
\begin{aligned}
& f\left(x_{1}, 0,0\right) \equiv 0 \Leftrightarrow g_{11}\left(x_{1}, 0,0\right) \equiv 0, \\
& f\left(0, x_{2}, 0\right) \equiv 0 \Leftrightarrow g_{22}\left(0, x_{2}, 0\right) \equiv 0, \\
& f\left(0,0, x_{3}\right) \equiv 0 \Leftrightarrow g_{33}\left(0,0, x_{3}\right) \equiv 0 .
\end{aligned}
$$


Then

$$
\begin{aligned}
x_{1}^{2} g_{11}\left(x_{1}, x_{2}, x_{3}\right) & =x_{1}^{2}\left(g_{11}\left(x_{1}, x_{2}, x_{3}\right)-g_{11}\left(x_{1}, 0,0\right)\right) \\
& =x_{1}^{2}\left(x_{2} h_{1}\left(x_{1}, x_{2}, x_{3}\right)+x_{3} h_{2}\left(x_{1}, x_{2}, x_{3}\right)\right),
\end{aligned}
$$

hence $x_{1}^{2} g_{11} \in\left\langle x_{1} x_{2}, x_{1} x_{3}, x_{2} x_{3}\right\rangle$. Using the same argument we get that $x_{2}^{2} g_{22}$ and $x_{3}^{2} g_{33}$ belong to $\left\langle x_{1} x_{2}, x_{1} x_{3}, x_{2} x_{3}\right\rangle$.

Theorem 4 [P]. Let $f \in \mathfrak{m}(n)^{\infty}$. Then there exists $g \in \mathfrak{m}(n)^{\infty}$ and $h \in \mathfrak{m}(n)^{\infty}$ with $g(x)>0$ for $x \neq 0$ such that

$$
f=g h \text {. }
$$

Corollary 5. If $I$ is an ideal of $\mathbf{R}[x]$ then $\widehat{I} \cap \mathfrak{m}(n)^{\infty}=\widehat{I} \mathfrak{m}(n)^{\infty}$.

Proof. One contention is obvious. For the other let $f \in \mathfrak{m}(n)^{\infty} \cap \widehat{I}$; then $f=g h$ as in the previous lemma. Since $\left.f\right|_{S} \equiv 0$ we get $\left.h\right|_{S} \equiv 0$ and hence $f \in \mathfrak{m}(n)^{\infty} \widehat{I}$, where $S=z(I)$.

Theorem 6 (Artin-Rees). Let $A=\mathbf{R}[[x]], x=\left(x_{1}, \ldots, x_{n}\right)$, be the formal power series ring, with $M$ its maximal ideal and $I$ an ideal of $A$. Then there exists $k$ such that for $m \geq k$

$$
I \cap \mathbf{M}^{m}=\mathbf{M}^{m-k}\left(I \cap \mathbf{M}^{k}\right) .
$$

We denote the minimal $k$ with such property by $\mathscr{A}(I)$.

Examples. (1) If $I=\left\langle x_{1}, \ldots, x_{s}\right\rangle$, then $\mathscr{A}(I)=1$.

(2) If $I=\left\langle x_{i} x_{j}\right\rangle_{1<i \leq s}^{n-t+1 \leq j \leq n}, s+t \leq n$, then $\mathscr{A}(I)=2$.

(3) If $I=\left\langle x_{1}^{2}-x_{2}^{3}\right\rangle$, then $\mathscr{A}(I)=2$.

Consider $\pi: \mathscr{E}(n) \rightarrow \mathbf{R}[[x]]$, the canonical Taylor series map, and let $I$ be an ideal in $\mathbf{R}[[x]]$ generated by polynomials. From Theorem 6 we get for $m \geq k$,

$$
I \cap \mathfrak{m}^{m}=\mathfrak{m}^{m-k}\left(I \cap \mathfrak{m}^{k}\right)+\mathfrak{m}^{\infty} \cap I,
$$

where $\mathfrak{m}$ is the maximal ideal of $\mathscr{E}(n)$ and $I$ is now viewed as an ideal in $\mathscr{E}(n)$.

Corollary 7. (1) For $I=\left\langle x_{1}, \ldots, x_{s}\right\rangle$ we get $I \cap \mathfrak{m}^{l+1}=\mathfrak{m}^{l} I+\mathfrak{m}^{\infty} \cap I \forall l$.

(2) For $I=\left\langle x_{i} x_{j}\right\rangle_{1 \leq i \leq s}^{n-t+1 \leq j \leq n}$ we get $I \cap \mathfrak{m}^{l+2}=\mathfrak{m}^{l} I+\mathfrak{m}^{\infty} \cap I \forall l$, where $s+t \leq n$.

(3) For $I=\left\langle x_{1}^{2}-x_{2}^{3}\right\rangle$ we get $I \cap \mathfrak{m}^{l+2}=\mathfrak{m}^{l} I+\mathfrak{m}^{\infty} \cap I \forall l$.

Lemma 8. In each of the above cases $I=\widehat{I}$ and hence $\mathfrak{m}^{\infty} \cap I=\mathfrak{m}^{\infty} I \subset \mathfrak{m}^{l} I$. Then we get the following equalities:

(1) $I \cap \mathfrak{m}^{l+1}=I \mathfrak{m}^{l} \forall l$.

(2) $I \cap \mathfrak{m}^{l+2}=I \mathfrak{m}^{l} \forall l$.

(3) $I \cap \mathfrak{m}^{l+2}=I \mathfrak{m}^{l} \forall l$.

Proof. The first two cases are easy consequences of Hadamard's lemma. For the third case $(n=2)$ let $\phi(x, y)=\left(x, x^{2}-y^{3}\right)$. Then by the Malgrange Preparation Theorem for $f \in \mathfrak{m}(2)$ we get

$$
f(x, y)=h_{0}\left(x, x^{2}-y^{3}\right)+y h_{1}\left(x, x^{2}-y^{3}\right)+y^{2} h_{2}\left(x, x^{2}-y^{3}\right) .
$$


If $S=\left\{(x, y) \mid x^{2}-y^{3}=0\right\}$ and $\left.f\right|_{S} \equiv 0$ we get $0=h_{0}(x, 0)+y h_{1}(x, 0)+$ $y^{2} h_{2}(x, 0)$ if $x^{2}-y^{3}=0$, hence $0=h_{0}\left(x^{3}, 0\right)+x^{2} h_{1}\left(x^{3}, 0\right)+x^{4} h_{2}\left(x^{3}, 0\right)$ and $\pi\left(h_{0}(x, 0)\right)=\pi\left(h_{1}(x, 0)\right)=\pi\left(h_{2}(x, 0)\right)=0$. Then

$$
\begin{aligned}
f= & \left(h_{0}\left(x, x^{2}-y^{3}\right)-h_{0}(x, 0)\right)+\left(h_{1}\left(x, x^{2}-y^{3}\right)-h_{1}(x, 0)\right) y \\
& +\left(h_{2}\left(x, x^{2}-y^{3}\right)-h_{2}(x, 0)\right) y^{2}+\eta(x) \\
= & \left(x^{2}-y^{3}\right) g(x, y)+\eta(x), \quad \eta \in \mathfrak{m}(1)^{\infty} .
\end{aligned}
$$

Finally, since $\left.f\right|_{S} \equiv 0 \Rightarrow \eta(x) \equiv 0$ for $x^{2}-y^{3}=0$, it follows that $\eta \equiv 0$ and $\widehat{J} \subset J$. The other contention is obvious.

Proposition 9. Let $I$ be an ideal of $\mathbf{R}[x]$ and consider $I$ as an ideal of $\mathscr{E}(n)$. Hence $I=\widehat{I}$ if and only if $\pi(I)=\pi(\widehat{I})$ and $\widehat{I}$ is finitely generated in $\mathscr{E}(n)$.

Proof. $(\Rightarrow)$ Obvious.

$(\Leftarrow)$ Our equality is equivalent to $I+\mathfrak{m}(n)^{\infty}=\widehat{I}+\mathfrak{m}(n)^{\infty}$; if we intersect with $\widehat{I}$ we get $I+\mathfrak{m}(n)^{\infty} \cap \widehat{I}=\widehat{I}$. Since $\mathfrak{m}(n)^{\infty} \cap \widehat{I}=\mathfrak{m}(n)^{\infty} \widehat{I}$ and $\widehat{I}$ is a finitely generated $\mathscr{E}(n)$-module, by Nakayama's lemma we get $I=\widehat{I}$.

Observation. By Theorem 2 of $[\mathrm{K}], \widehat{I}$ is a finitely generated ideal if and only if $z(I)$ is a coherent algebraic set.

Lemma 10. Let $I=\left\langle f_{1}, \ldots, f_{s}\right\rangle$ be polynomials in $\mathbf{R}[[x]]$, let $S=z(I)$ be their common zeros, and suppose $I$ is radical. Consider $\widetilde{I}=\left\langle\tilde{f}_{1}, \ldots, \tilde{f}_{s}, t\right\rangle$ in $\mathbf{R}[[x, t]]$, where $\tilde{f}_{i}\left(x_{1}, \ldots, x_{n}, t\right)=f_{i}\left(x_{1}, \ldots, x_{n}\right)$. Then $\hat{\tilde{I}}=\widetilde{I}$ and $\mathscr{A}(I)=$ $\mathscr{A}(\widetilde{I})$.

Proof. It is clear that $z(\widetilde{I})=S \times\{0\}$. Let

$$
\phi:\left\{g \in \mathscr{E}(n+1)|g|_{S \times\{0\}}=0\right\} \rightarrow\left\{f \in \mathscr{E}(n)|f|_{S}=0\right\} \times\langle t\rangle \mathscr{E}(n+1)
$$

be given by $\phi(g)=\left(g\left(x_{1}, \ldots, x_{n}, 0\right), g-g\left(x_{1}, \ldots, x_{n}, 0\right)\right)$. This map is clearly an isomorphism and hence

$$
\hat{\tilde{I}} \simeq \hat{I} \times\langle t\rangle \mathscr{E}(n+1)=I \times\langle t\rangle \mathscr{E}(n+1) .
$$

Similarly, $\tilde{I}=\left\langle\tilde{f}_{1}, \ldots, \tilde{f}_{s}, t\right\rangle \simeq I \times\langle t\rangle \mathscr{E}(n+1)$. Then $\hat{\tilde{I}}=\tilde{I}$ and using Theorem 6 with $\mathfrak{m}(n+1)$ instead of $\mathfrak{m}(n)$ we have $\widetilde{I} \cap \mathfrak{m}(n+1)^{m}=$ $\mathfrak{m}(n+1)^{m-k}\left(\tilde{I} \cap \mathfrak{m}(n+1)^{k}\right)$.

Theorem 11. Let I be a radical ideal. If $\mathfrak{m}(n)^{m} \cap I \subset I\langle d f\rangle$ and $I \cap \mathfrak{m}(n)^{k}$ is finitely generated, where $\mathscr{A}(I)=k$, then $f$ is m-determined relative to $G_{S}$, where $S=z(I)$.

Proof. Let $t_{0} \in \mathbf{R}$ be fixed, $g$ a germ with $\left.\left.g\right|_{S} \equiv f\right|_{S}$, and $j^{m} f(0)=j^{m} g(0)$. Consider the map $F:\left(\mathbf{R}^{n} \times \mathbf{R},\left(0, t_{0}\right)\right) \rightarrow \mathbf{R}$ given by $F(x, t)=F_{t}(x)=$ $(1-t) f(x)+\operatorname{tg}(x)$.

We will show that $F_{t}$ is $G_{S}$-equivalent to $F_{t_{0}}$ if $t \sim t_{0}$.

By Theorem 2 it is enough to find $h:\left(\mathbf{R}^{n} \times \mathbf{R}, 0 \times t_{0}\right) \rightarrow \mathbf{R}^{n}$ such that

(I) $\sum_{i=1}^{n} \frac{\partial F}{\partial x_{i}}(x, t) h_{i}(x, t)+\frac{\partial F}{\partial t}(x, t)=0$,

(II) $h_{i}(x, t)=0$ for $t \sim t_{0}, x \sim 0$ in $S$.

Let $N=\left\{\omega \in \mathscr{E}(n+1)|\omega|_{S \times\left\{t_{0}\right\}}=0\right.$ and $\left.j^{m-1} \omega_{t}(0)=0, t \sim t_{0}\right\}$ and $K=\left\{\sum_{i=1}^{n} \frac{\partial F}{\partial x_{i}}(x, t) h_{i}(x, t) \mid h_{i}\right.$ as in II $\}$. 
By Lemma 10, $N$ is a finitely generated module.

If we can show that $N \subset K$, we have $\partial F / \partial t=g-f \in K$ and we obtain conditions (I) and (II).

Letting $h \in N$, we can write $h(x, t)=h(x, t)-h\left(x, t_{0}\right)+h\left(x, t_{0}\right)$. It is clear that $h(x, t)-h\left(x, t_{0}\right) \in \mathfrak{m}(n+1) N$. On the other hand, $h\left(x, t_{0}\right) \in$ $\mathfrak{m}(n)^{m} \cap I \subset I\langle d f\rangle ;$ then

$$
h\left(x, t_{0}\right)=\sum_{i=1}^{n} \frac{\partial f}{\partial x_{i}}(x) \eta_{i}(x), \quad \eta_{i} \in I \forall i .
$$

By hypothesis,

$$
g-f \in \mathfrak{m}(n)^{m+1} \cap I=\mathfrak{m}(n)^{m+1-k}\left(\mathfrak{m}(n)^{k} \cap I\right),
$$

hence $\left(\partial g / \partial x_{i}-\partial f / \partial x_{i}\right)(x) \eta_{i}(x) \in N$ and

$$
h\left(x, t_{0}\right)=\sum_{i=1}^{n} \frac{\partial F}{\partial x_{i}}(x, t) \eta_{i}(x)-t \sum_{i=1}^{n}\left(\frac{\partial g}{\partial x_{i}}-\frac{\partial f}{\partial x_{i}}\right)(x) \eta_{i}(x)
$$

is an element of $K+\mathfrak{m}(n+1) N$. Thus, $N \subset K+\mathfrak{m}(n+1) N$, which by Nakayama's lemma implies $N \subset K$.

Notation. Let $z \in J_{0}^{q}(n, 1)$ be the space of $q$-jets which send $\overline{0}$ to 0 , and $f$ a representative of $z$. Let

$$
J_{0}^{q}(f, S, n)=\left\{j^{q} g(0) \mid g-f \in J\right\},
$$

and let $\bar{\pi}_{q}: f+J \rightarrow J_{0}^{q}(f, S, n)$ and $\bar{\pi}_{q}: J \rightarrow J_{0}^{q}(0, S, n):=J_{S}^{q}(n)$ be the restrictions of the canonical map $\pi_{q}: \mathscr{E}(n) \rightarrow J^{q}(n)$.

Finally, let $G_{S}^{q}=\left\{j^{q} h(0) \mid h \in G_{S}\right\}$ and $z G_{S}^{q}$ be the orbit of $z$.

Proposition 12. Let I be the ideal of $\mathbf{R}[x]$. If $\overline{0} \in S=z(I)$ then $G_{S}^{q}$ is a Lie group.

Proof. We shall show that

$$
\left.G_{S}^{q}=\left\{j^{q}\left(\mathrm{Id}+\left(h_{1}, \ldots, h_{n}\right)\right) \mid h_{i} \in \widehat{I}\right)\right\} \cap G^{q},
$$

where $G^{q}=G_{\{\overline{0}\}}^{q}$.

Let $\sigma=j^{q} \phi \in G_{S}^{q}$, where $\phi=\left(\phi_{1}, \ldots, \phi_{n}\right)$; then $\left.\phi\right|_{S}=$ Id. If we write $\phi=\mathrm{Id}+(\phi-\mathrm{Id})$, we clearly have that $h_{i}=\phi_{i}-x_{i} \in \widehat{I}$. The other contention is obvious.

Hence $G_{S}^{q}$ is a closed subgroup of the Lie group $G^{q}$.

Observation. $T_{\mathrm{Id}} G_{S}^{q}=j^{q}(\widehat{I} \times \cdots \times \widehat{I})$.

Lemma 13. $\bar{\pi}_{q}^{-1}\left(T_{z} z G_{S}^{q}\right)=\widehat{I}\langle d f\rangle+\widehat{I} \cap \mathfrak{m}(n)^{q+1}$.

Proof. Let $\beta \in T_{\mathrm{Id}} G_{S}^{q}$ be a tangent vector, $\beta=j^{q} \beta^{\prime}$. For $t \in R$ we define $\delta_{t}=$ Id $+t \beta^{\prime}$. If we consider $\pi_{q} \circ \delta_{t}:(-\varepsilon, \varepsilon) \rightarrow G_{S}^{q}$, then $\beta=\left.\frac{\partial}{\partial t}\left(\pi_{q} \circ \delta_{t}\right)\right|_{t=0}$. On the other hand,

$$
\left.\frac{\partial}{\partial t}\left(z \cdot\left(\pi_{q} \circ \delta_{t}\right)\right)\right|_{t=0}=\left.\frac{\partial}{\partial t}\left(\pi_{q}\left(f \circ \delta_{t}\right)\right)\right|_{t=0}=\pi_{q}\left(\sum_{i=1}^{n} \frac{\partial f}{\partial x_{i}} \beta_{i}^{\prime}\right),
$$

where $\beta_{i}^{\prime} \in \widehat{I}$. 
Then $T_{z} z G_{S}^{q}=\bar{\pi}_{q}(\langle d f\rangle \widehat{I})$ and hence $\bar{\pi}_{q}^{-1}\left(T_{z} z G_{S}^{q}\right)=\langle d f\rangle \widehat{I}+\mathfrak{m}(n)^{q+1} \cap \widehat{I}$.

Lemma 14. Let $q \geq 0$ and $z \in J_{0}^{q}(n, 1)$ such that $z=j^{q} f$, and let $l \leq q$. If $z$ is l-determined, then

$$
\widehat{I} \cap \mathfrak{m}(n)^{l+1} \subset \widehat{I}\langle d f\rangle+\mathfrak{m}(n)^{q+1} \cap \widehat{I} .
$$

Proof. Let $A=\left\{z^{\prime} \in J_{S}^{q}(n) \mid \pi_{q, l}\left(z^{\prime}\right)=\pi_{q, l}(z)\right\}$ where $\pi_{q, l}: J_{0}^{q}(n) \rightarrow J_{0}^{l}(n)$ is the canonical projection. Since $A$ is an affine space, it follows that $T_{z} A=$ $\bar{\pi}_{q}\left(\widehat{I} \cap \mathfrak{m}(n)^{l+1}\right)$. By hypothesis we have $A \subset z G_{S}^{l}$, hence $T_{z} A \subset T_{z} z G_{S}^{l}$ and $\bar{\pi}_{q}\left(\widehat{I} \cap \mathfrak{m}(n)^{l+1}\right) \subseteq \bar{\pi}_{q}(\langle d f\rangle \widehat{I})$. As before we get $\widehat{I} \cap \mathfrak{m}(n)^{l+1} \subseteq \widehat{I}\langle d f\rangle+\mathfrak{m}^{q+1}(n) \cap$ $\widehat{I}$.

Theorem 15. Let $f$ be an m-determined germ relative to $G_{S}$, where $S=z(I)$, $I$ radical, and $k=\mathscr{A}(I)$. Then

$$
I \cap \mathfrak{m}(n)^{m+1} \subset I\langle d f\rangle \quad \text { for } m \geq k .
$$

Proof. Since $f$ is m-determined relative to $G_{S}, \bar{\pi}_{m+1} f$ is $m$-determined relative to $G_{S}^{m+1}$ and, using Lemma 14 with $k=m$ and $q=m+1$, we obtain

$$
\widehat{I} \cap \mathfrak{m}(n)^{m+1} \subset \widehat{I}\langle d f\rangle+\mathfrak{m}(n)^{m+2} \cap \widehat{I}
$$

but $\mathfrak{m}(n)^{m+2} \cap \widehat{I}=\mathfrak{m}(n)\left(\mathfrak{m}(n)^{m+1} \cap \widehat{I}\right)$ and by Nakayama's lemma we obtain

$$
\widehat{I} \cap \mathfrak{m}(n)^{m+1} \subseteq \widehat{I}\langle d f\rangle .
$$

Joining Theorems 11 and 15 we obtain

Theorem 16. Let $f \in \mathfrak{m}(n), I=\left\langle f_{1}, \ldots, f_{s}\right\rangle$ be a radical ideal in $R[x]$, and $S$ be the set of common zeros. Suppose $\mathscr{A}(I)=k$ and $\widehat{I} \cap \mathfrak{m}(n)^{k}$ is finitely generated. Then $f$ is finitely determined relative to $G_{S}$ if and only if there exists $l$ such that $\mathfrak{m}(n)^{l} \cap I \subset I\langle d f\rangle$.

Observation. Let $I$ be the ideal of $\mathscr{E}(n)$ and suppose $\pi\left(I \cap \mathfrak{m}(n)^{k}\right)$ is generated by $\left\{h_{1}, \ldots, h_{s}\right\}$. We let $f_{i} \in \mathscr{E}(n)$ be such that $\pi\left(f_{i}\right)=h_{i}$ for $1 \leq i \leq s$ and we write $f_{i}=g_{i}+\xi_{i}$, where $g_{i} \in I \cap \mathfrak{m}(n)^{k}$ and $\xi_{i} \in \mathfrak{m}(n)^{\infty}$. Then we have

$$
I \cap \mathfrak{m}(n)^{k}=\left\langle g_{1}, \ldots, g_{s}\right\rangle+\mathfrak{m}(n)^{\infty} \cap I .
$$

Theorem 17. If $I=\widehat{I}$, the following three assertions are equivalent:

(1) $I \cap \mathfrak{m}(n)^{k}$ is a finitely generated ideal of $\mathscr{E}(n)$,

(2) $I \cap \mathfrak{m}(n)^{k}=\left\langle g_{1}, \ldots, g_{s}\right\rangle$,

(3) $\left\langle g_{1}, \ldots, g_{s}\right\rangle \supset I \cap \mathfrak{m}(n)^{\infty}$.

Proof. (1) $\Rightarrow(2)$. Since $I \mathfrak{m}(n)^{\infty}=\left(I \cap \mathfrak{m}(n)^{\infty}\right) \mathfrak{m}(n)^{\infty}$, we have $I \cap \mathfrak{m}(n)^{k}=$ $\left\langle g_{1}, \ldots, g_{s}\right\rangle+\mathfrak{m}(n)^{\infty} I \supset\left\langle g_{1}, \ldots, g_{s}\right\rangle+\mathfrak{m}(n)^{\infty}\left(I \cap \mathfrak{m}(n)^{k}\right) \supset\left\langle g_{1}, \ldots, g_{s}\right\rangle+$ $\mathfrak{m}(n)^{\infty}\left(I \cap \mathfrak{m}(n)^{\infty}\right)=\left\langle g_{1}, \ldots, g_{s}\right\rangle+\mathfrak{m}(n)^{\infty} I$.

Then $I \cap \mathfrak{m}(n)^{k}=\left\langle g_{1}, \ldots, g_{s}\right\rangle+\mathfrak{m}(n)^{\infty}\left(I \cap \mathfrak{m}(n)^{k}\right)$ and by Nakayama's lemma we get $I \cap \mathfrak{m}(n)^{k}=\left\langle g_{1}, \ldots, g_{s}\right\rangle$.

(2) $\Rightarrow(3)$. Obvious.

(3) $\Rightarrow(1)$. From $(*)$ we get $I \cap \mathfrak{m}(n)^{k}=\left\langle g_{1}, \ldots, g_{s}\right\rangle$.

Lemma 18. Let $\left\{p_{j}=x_{1}^{i_{j}^{1}} \cdots x_{n}^{i_{j}^{n}}\right\}_{j=1}^{s}$ be monomials with $0 \leq i_{1}^{k} \leq 1, \ldots$, $0 \leq i_{n}^{k} \leq 1$ and $\sum_{k=1}^{n} i_{j}^{k}=\alpha>0$. Then 
(1) $I=\widehat{I}$,

(2) $\mathfrak{m}(n)^{m} \cap I=\mathfrak{m}(n)^{m-\alpha} I \quad \forall m \geq \alpha$,

where $I$ is the ideal generated by the polynomials $p_{j}$.

Theorem 19. Let $f$ be a germ finitely determined on the right, $\left\{p_{j}\right\}_{j=1}^{s}$ monomials as in the previous lemma, and $S=z(I)$. Then $f$ is finitely determined relative to $G_{S}$.

Proof. We know there exists an $l$ such that $\mathfrak{m}(n)^{l} \subset\langle d f\rangle$, hence $\mathfrak{m}(n)^{l} I \subset$ $I\langle d f\rangle$. If we set $l=m-\alpha$, we will have $\mathfrak{m}(n)^{m} \cap I \subset I\langle d f\rangle$. Applying Theorem 11 we finish.

Theorem 20. Let $I=\left\langle p_{j}\right\rangle_{j=1}^{s}, p_{j}$ monomials of degree $\alpha$ as in Lemma 19. Suppose that $f$ is finitely determined relative to $G_{S}$, where $S=z(I)$, and that

$$
W_{i}=\overline{\left(\bigcap_{\substack{j=1 \\ j \neq 1}}^{k} z\left(p_{j}\right) z(I)-z\left(p_{i}\right)\right)} \supset \bigcap_{j=1}^{k} z\left(p_{j}\right)=z(I) \quad \forall_{i} .
$$

Then $f$ is finitely determined on the right.

Proof. We know there exists an $m$ such that $\mathfrak{m}(n)^{m} \cap I \subset I\langle d f\rangle$.

Let $x \in \mathfrak{m}(n)^{2(m-\alpha)}$ and put $x=y y^{\prime}$ with $y, y^{\prime}$ in $\mathfrak{m}(n)^{m-\alpha}$. Then

$$
y p_{i} \in \mathfrak{m}(n)^{m-\alpha} I=\mathfrak{m}(n)^{m} \cap I \subset I\langle d f\rangle,
$$

hence

$$
\begin{aligned}
y p_{i} & =\sum_{i=1}^{s} \frac{\partial f}{\partial x_{j}} h_{j} \quad\left(\text { where } h_{j} \in I=\left\langle p_{1}, \ldots, p_{s}\right\rangle\right) \\
& =\sum_{j=1}^{s} \sum_{k=1}^{s} h_{k}^{j} p_{k} \frac{\partial f}{\partial x_{j}}=p_{i} \sum_{j=1}^{s} h_{i}^{j} \frac{\partial f}{\partial x_{j}}+\sum_{j=1}^{s} \sum_{k \neq i} h_{k}^{j} p_{k} \frac{\partial f}{\partial x_{j}} .
\end{aligned}
$$

If we denote $\phi=y-\sum_{j=1}^{s} h_{i}^{j} \partial f / \partial x_{j}$, we get

$$
p_{i} \cdot \phi=\sum_{j=1}^{s} \sum_{j \neq i} h_{k}^{j} p_{k}\left(\frac{\partial f}{\partial x_{j}}\right) .
$$

Hence $\phi$ vanishes in $W_{i}$, and by hypothesis $\phi \in I$. If we denote $\gamma=$ $\sum_{j=1}^{s} h_{i}^{j}\left(\partial f / \partial x_{j}\right)$ we obtain $p_{i} y=p_{i}(\phi+\gamma)$, so $y=\phi+\gamma$ and

$$
x=y y^{\prime}=\phi y^{\prime}+\gamma y^{\prime} \text {. }
$$

Since $\phi \in I, \phi y^{\prime} \in I \mathfrak{m}(n)^{m-\alpha}=\mathfrak{m}(n)^{m} \cap I \subset I\langle d f\rangle$, and $\gamma y^{\prime} \in\langle d f\rangle$, it follows that $x \in\langle d f\rangle$. We have shown that $\mathfrak{m}(n)^{2(m-\alpha)} \subset\langle d f\rangle$, therefore $f$ is finitely determined on the right.

Example. $I=\left\langle x_{1} x_{2}, x_{3} x_{4}\right\rangle$.

Definition 21. Let $f: \mathbf{R}^{n}, 0 \rightarrow \mathbf{R}$ be an analytic germ which is finitely determined on the right. Then

$$
l(f)=\min \left\{k \mid\langle d f\rangle \supset \mathbf{M}^{k} \text { and } \mathbf{M}\langle d f\rangle \not \supset \mathbf{M}^{k}\right\} .
$$


Proposition 22. Consider $I=\langle d f\rangle$ in $\mathbf{R}[[x]]$ and suppose $\mathscr{A}(I)=s$. Then we have $l(f)=s$.

Proof. From the definition of $l=l(f)$ it is clear that $\mathbf{M}^{l+r}=\langle d f\rangle \cap \mathbf{M}^{l+r} \forall r \geq 0$ and $\left(\langle d f\rangle \cap \mathbf{M}^{l}\right) \mathbf{M}^{r}=\mathbf{M}^{l+r} \forall r \geq 0$, hence

$$
\langle d f\rangle \cap \mathbf{M}^{l+r}=\left(\langle d f\rangle \cap \mathbf{M}^{l}\right) \mathbf{M}^{r} \quad \forall r \geq 0 .
$$

Thus, $l \geq s$. If $l>s$ we have $\left(\langle d f\rangle \cap \mathbf{M}^{s}\right) \mathbf{M}^{r}=\langle d f\rangle \cap \mathbf{M}^{s+r} \forall r \geq 0$.

In particular for $r=1$ we get $\left(\langle d f\rangle \cap \mathbf{M}^{s}\right) \mathbf{M}=\langle d f\rangle \cap \mathbf{M}^{s+1}$ and $\mathbf{M}^{l} \subset$ $\langle d f\rangle \cap \mathbf{M}^{s+1}=\left(\langle d f\rangle \cap \mathbf{M}^{s}\right) \mathbf{M} \subset\langle d f\rangle \mathbf{M}$, but this contention contradicts the choice of $l=l(f)$.

Ideals of Lojasiewicz. Let $C^{\infty}(\Omega, \mathbf{R})$ be the algebra of smooth functions from an open set $\Omega$ in $\mathbf{R}^{n}$ to $\mathbf{R}$. We let $X$ be a closed subset of $\mathbf{R}^{n}$.

Definition 23. (1) We say that a function $f$ satisfies a Lojasiewicz inequality for $X$ if for every compact subset $K$ of $\Omega$ there exist constants $C>0, \alpha \geq 0$ such that

$$
|f(x)| \geq C d(x, X)^{\alpha} \quad \forall x \in K .
$$

(2) An ideal $I$ of $C^{\infty}(\Omega, \mathbf{R})$ is a Lojasiewicz ideal if there exists a map in $I$ with the Lojasiewicz property for $X=z(I)$, the set of common zeros of $I$.

(3) $J_{k}(I)$ is the ideal generated by $I$ and all the $k \times k$ minors of the matrix $\left(\partial f_{i} / \partial x_{j}\right), 1 \leq j \leq k, 1 \leq j \leq n$, where $f_{1}, \ldots, f_{k}$ belong to $I$.

Proposition 24 (Tougeron). If $I=\left\langle\varphi_{1}, \ldots, \varphi_{p}\right\rangle$ and $J_{p}(I)$ is a Lojasiewicz ideal, then

1. I itself is a Lojasiewicz ideal.

2. If $f$ is flat on $z\left(J_{p}(I)\right)$ and $\left.f\right|_{z(I)} \equiv 0$, then $f$ belongs to $I$.

Example. $I=\left\langle x^{2}+y^{2}\right\rangle, J_{1}(I)=\langle x, y\rangle$. Hence $z\left(J_{1}(I)\right)=\{\overline{0}\}$ and $\mathfrak{m}(n)^{\infty} \subset$ $I$. That means that for $f \in \mathfrak{m}(n)^{\infty}$ there exists $g_{1}$ such that $f=\left(x^{2}+y^{2}\right) g_{1}$.

Corollary 25. If we consider our local case,

$$
I=\left\langle\varphi_{1}, \ldots, \varphi_{p}\right\rangle \text { and } z\left(J_{p}\left(\varphi_{1}, \ldots, \varphi_{p}\right)\right)=\{\overline{0}\},
$$

where $\varphi_{i}$ are analytic, then

(1) $\mathfrak{m}(n)^{\infty} \cap \widehat{I}=\mathfrak{m}(n)^{\infty} \widehat{I}=\mathfrak{m}(n)^{\infty} I=\mathfrak{m}(n)^{\infty} \cap I$,

(2) $\widehat{I}$ is finitely generated.

Proof. The first part is a direct consequence of the last proposition.

For the second part let $I=\left\langle\varphi_{1}, \ldots, \varphi_{p}\right\rangle$. Now $\pi(\widehat{I})$ is finitely generated, hence we have $\pi(\widehat{I})=\left\langle h_{1}, \ldots, h_{s}\right\rangle, h_{i} \in \mathbf{R}[[x]], 1 \leq i \leq s$. Let $g_{i} \in \widehat{I}$ with $\pi\left(g_{i}\right)=h_{i}, \quad 1 \leq i \leq s$. Therefore $\widehat{I}=\left\langle g_{1}, \ldots, g_{s}\right\rangle+\widehat{I} \cap \mathfrak{m}(n)^{\infty}$. We can suppose that $\left\{\varphi_{1}, \ldots, \varphi_{p}\right\} \subset\left\{g_{1}, \ldots, g_{s}\right\}$. Since $\hat{I} \cap \mathfrak{m}(n)^{\infty} \subset I$ we get $\widehat{I}=\left\langle g_{1}, \ldots, g_{s}\right\rangle$.

Theorem 26. Suppose $I=\left\langle f_{1}, \ldots, f_{p}\right\rangle$ is an ideal of analytic maps and that

(1) $J_{p}(I)$ is a Lojasiewicz ideal.

(2) $z\left(J_{p}(I)\right)=\{\overline{0}\}$.

(3) $\widehat{I} \cap \mathfrak{m}(n)^{k}$, where $k=\mathscr{A}(\pi(\widehat{I}))$ is finitely generated.

If $\mathfrak{m}(n)^{m} \cap \widehat{I} \subset \widehat{I}\langle d f\rangle$, then $f$ is $m$-determined relative to $G_{S}$, where $S=$ $z(I)$.

Proof. (1) $\exists k$ with $\pi(\widehat{I}) \cap \mathbf{M}(n)^{m}=\mathbf{M}(n)^{m-k}\left(\pi(\widehat{I}) \cap \mathbf{M}^{k}(n)\right)$. 
(2) $\pi^{-1}\left(\pi(\widehat{I}) \cap \mathbf{M}(n)^{m}\right)=\widehat{I} \cap \mathfrak{m}^{m}(n)+\mathfrak{m}(n)^{\infty}$.

Let $f \in \pi^{-1}\left(\pi(\widehat{I}) \cap \mathbf{M}(n)^{m}\right)$; then $\pi(f) \in \pi(\widehat{I}) \cap \mathbf{M}(n)^{m}$. Hence there exists $g \in \widehat{I}$ with $\pi(g)=\pi(f)$ and $\pi(g) \in \mathbf{M}(n)^{m}$. Hence $g \in \widehat{I} \cap m(n)^{m}$ and $f \in \widehat{I} \cap m(n)^{m}+m(n)^{\infty}$.

Conversely let $g \in \widehat{I} \cap m(n)^{m}+m(n)^{\infty}$. Then $\pi(g) \in \pi(\widehat{I}) \cap \pi\left(m(n)^{m}\right)=$ $\pi(\widehat{I}) \cap \mathbf{M}(n)^{m}$.

(3) $\pi^{-1}\left(\mathbf{M}(n)^{m-k}\left(\pi(\widehat{I}) \cap \mathbf{M}(n)^{m}\right)\right)=m(n)^{m-k}\left(\widehat{I} \cap m(n)^{k}\right)+\mathfrak{m}(n)^{\infty}$. This is done in a similar way to (2).

From (1) we get

$$
\widehat{I} \cap m(n)^{m}+m(n)^{\infty}=m(n)^{m-k}\left(\widehat{I} \cap m(n)^{k}\right)+m(n)^{\infty},
$$

and if we intersect each member of the equality with $\widehat{I}$, we get

$$
\begin{aligned}
\widehat{I} \cap m(n)^{m} & =m(n)^{m-k}\left(\widehat{I} \cap m(n)^{k}\right)+\widehat{I} \cap m(n)^{\infty} \\
& =m(n)^{m-k}\left(\widehat{I} \cap m(n)^{k}\right)+\widehat{I} m(n)^{\infty} \\
& =m(n)^{m-k}\left(\widehat{I} \cap m(n)^{k}\right) \quad \forall m \geq k .
\end{aligned}
$$

Since $\widehat{I} \cap m(n)^{k}$ is finitely generated, so is $\widehat{I} \cap m(n)^{m} \quad \forall m \geq k$. We now proceed as in Theorem 11 .

Corollary 27. Let $f \in m(n)^{2}$ be a finitely determined analytic map and let I be the ideal generated by $f$. If $\widehat{I} \cap m(n)^{k}$ is finitely generated, where $k$ is as in (3) of the last theorem, then $f$ is finitely determined relative to $G_{S}$, where $S=f^{-1}(0)$.

Proof. Conditions (1) and (2) of the last theorem are obviously satisfied since there exists $l \in \mathbf{N}$ such that $\left(x_{1}^{2}+\cdots+x_{n}^{2}\right)^{l} \in J_{1}(f)$. Condition (3) is given by hypothesis. Now, since there exists $l$ with $m(n)^{l} \subset\langle d f\rangle$, we get

$$
\widehat{I} \cap m(n)^{m}=m(n)^{m-k}\left(\widehat{I} \cap m^{k}(n)\right) \subset \widehat{I}\langle d f\rangle
$$

for $m \geq k+l$.

We now use the last theorem to complete the proof.

\section{REFERENCES}

[A-M] M. Atiyah and I. Macdonald, Introduction to commutative algebra, Addison-Wesley, Reading, Mass., 1969.

[K] W. Kucharz, Analytic and differentiable functions vanishing on an algebraic set, Proc. Amer. Math. Soc. 102 (1988).

[M] J. Mather, Finitely determined map germs, Publ. Math. Inst. Hautes Études Sci. 35 (1968), 127-156.

[P-L] P. F. S. Porto and G. F. Loibel, Relative finite determinacy and relative stability of function germs, Bol. Soc. Brasil Mat. 9 (1978).

[P] P. Porto, On relative stability of function germs, Bol. Soc. Brasil Mat. 14 (1983).

Universidad Nacional Autonoma de Mexico, Ciudad Universitaria, Alvaro Obregon, 04510 Mexico City, Mexico 